\title{
EFIC-P539: MODULATING CORTICAL RESPONSES TO PAIN BY RESPIRATORY SINUS ARRHYTHMIA BIOFEEDBACK
}

Caroline Perchet ${ }^{1}$, Anne-Noëlle Heizmann², Amandine Rey ${ }^{1}$, Luis Garcia-Larrea ${ }^{1,3}$ and Stéphanie Mazza1,4

1: Central integration of pain, Center for Neuroscience of Lyon (Inserm U1028 \& University Claude Bernard Lyon 1), France

2 : 'Etude des Mécanismes cognitifs', University Lyon 2, Lyon, France

3: Centre d'évaluation et de traitement de la douleur, Hôpital Neurologique, Lyon, France

4: 'Hesper', University Lyon 1, Lyon, France

\section{AIM OF THE STUDY}

Cardiac Coherence (CC) is based on a respiratory sinus arrhythmia (RSA) biofeedback during a slow breathing training. Respiratory sinus arrhythmia characterises the variation in heart rate that occurs during each breathing cycle. RSA is known to have important regulatory functions and can be modulated using biofeedback procedures. It has been proposed that this helps stabilise autonomic tonus, which in turn might improve different disorders characterized by autonomic dysfunction $(1,2,3)$.

In this work we explore the effect of cardiac coherence on pain perception and cortical pain processing in healthy subjects after 2 weeks of daily training.

\section{METHODS}

\section{Baseline}

12 healthy subjects

Anxiety, depression, sleep questionnaires

$15 \mathrm{Nd}$ :YAP laser stimuli (baseline intensity for a VAS=4)

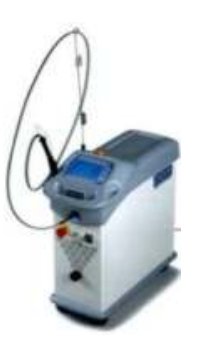

Training ( 2 weeks, $3 \mathrm{X}$ days, 5 minutes)

Cardiac Coherence' group (CCG) $(n=6 ; 4 F ; 22.3 y)$ HRV

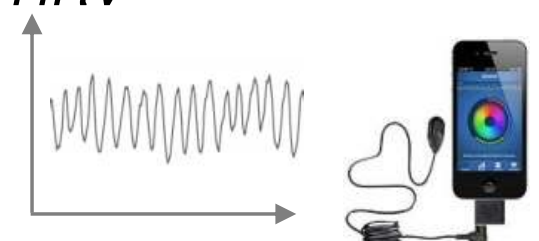

6 breaths $/ \mathrm{min}$

'Control' group (CG) (n=6; 4F; 23.7y) HRV

\section{Experimental sessions:}

\section{Session 1:}

15 laser stimuli (baseline intensity) $\rightarrow$ Resting EEG and LEPs recordings (32 channels) state

Session 2:

15 laser stimuli (baseline intensity) $\quad \rightarrow \rightarrow$ EEG and LEPs recordings (32 channels)

Cycle check

during normal breathing

\section{RESULTS}

\section{Pain perception}

LEPs

1- Baseline:

- No difference regarding level of anxiety, depression, or sleep parameters

- Same laser intensity to obtain a painful stimulation (VAS=4): (CG: 1.2 +/- $0.18 \mathrm{~J} / 4 \mathrm{~mm}$; CCG: $1.3+/-0.20$ $\mathrm{J} / 4 \mathrm{~mm}(\mathrm{p}=\mathrm{NS}))$

\section{2- Experimental sessions:}

Cardiac coherence (\% of time) Cardiac coherence (\% of time)

Session 1: 47.2+/-13.1

Session 2: $48.8+/-14.2$

Session1: $49.2+/-17.1$

Session2 : $86.8+/-14.7$

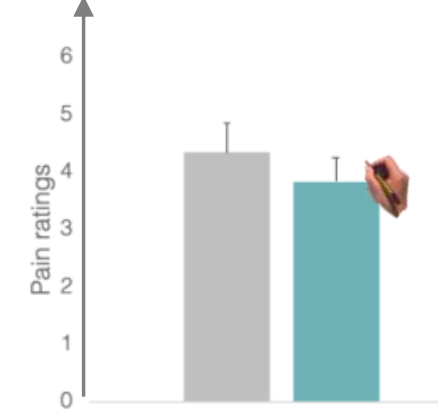

'Control' group

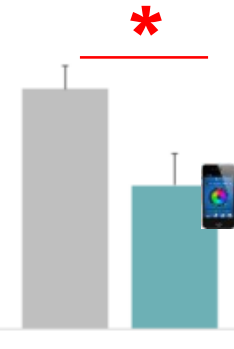

Resting state

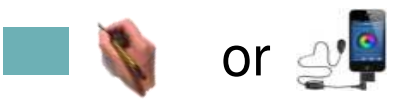

'Cardiac coherence' group

* $=p<0,05$

'Control' group:

- Normal breathing does not install a stable 'cardiac coherence' state and does not modulate pain ratings

\section{'Cardiac Coherence' group:}

- After 15 days of training subjects were able to install a stable 'cardiac coherence' state

- Cardiac coherence significantly reduced the pain ratings at a non painful level

\section{SUMMARY AND CONCLUSIONS}

Cardiac coherence is a respiratory biofeedback procedure which is non-invasive and cost-effective. Moreover this technique is easy to learn and perform and appears to modulate pain perception and acute cortical pain processing. Our results are encouraging enough to warrant further studies in healthy subjects (eg assessing descending pain modulation) as well as in patients with pain linked to autonomic dysfunction and stress. 